\title{
A Finite Element Analysis and Cyclic Load Experiment on an Additional Transcortical-Type Hole Formed Around The Proximal Femoral Nail System's Distal Locking Screw
}

Hong Man Cho ( $\nabla$ chm1228@hanmail.net )

Gwangju Veterans Hospital

Seung Min Choi

Inchon Veterans Hospital

JiYeon Park

Gwangju Veterans Hospital

\section{Young Lee}

Veterans Medical Research Institute, Veterans Health Service Medical Center

Jung Hyung Bae

ENS Corp

\section{Research Article}

Keywords: Intertrochanter fracture, Femur, Hole, Distal screw, Proximal femur nail, Complication

Posted Date: August 4th, 2021

DOI: https://doi.org/10.21203/rs.3.rs-737483/v1

License: (c) (i) This work is licensed under a Creative Commons Attribution 4.0 International License.

Read Full License

Version of Record: A version of this preprint was published at BMC Musculoskeletal Disorders on January 27th, 2022. See the published version at https://doi.org/10.1186/s12891-022-05006-4. 


\section{Abstract \\ Background}

A complication associated with the distal locking screw used in the proximal femoral nail (PFN) system is the formation of accidental additional holes. We hypothesized that an increase in stress around additional holes is a relevant factor contributing to fractures. This study aimed to evaluate stress changes in the cortical bone around additional screw holes using finite element analysis.

\section{Methods}

Proximal femoral nail PFN antirotation (PFNA)-II (Synthes, Solothurn, Switzerland) was inserted into a femur model. An additional 4.9-mm transcortical hole was made either anteriorly (anterior hole model) or posteriorly (posterior hole model) to the distal locking screw. Finite element analysis was used to calculate compression, tension, and load limits to investigate stress around additional holes with respect to the direction of screw penetration and degree of osteoporosis. The results were then compared with those of mechanical testing. A 31A-21 type intertrochanteric fracture was applied. As a control group, a model without additional holes (no-hole model) was developed. Repeated load-loading tests were performed on 10 model bones per model group.

\section{Results}

Tensile stress was significantly greater in the no-hole model when additional screw holes were present, and the anterior hole showed a higher maximum stress value than the posterior hole did, suggesting that the anterior hole was more susceptible to fracture. The change in tensile stress first appeared in the hole around the lateral cortical bone and proceeded to the medial side. Biomechanical testing showed that fractures around the distal locking screw occurred in 0 cases of the no-hole, 10 of anterior hole, and 9 of the posterior hole models.

\section{Conclusions}

During PFN surgery for intertrochanteric fracture, holes with distal locking screws fixed and removed at the anterior and posterior of the nail can be a risk factor for fractures in the surrounding area.

\section{Background}

The use of distal locking screws in the proximal femoral nail (PFN) system surgically stabilize femoral intertrochanteric fractures, provide length and rotational stability, and restrain the movement of the distal tip of the nail in broad medullary canals [1]. Although improvements in the PFN system have been made, complications due to the distal locking screw still occur [2]. One of the complications associated with the 
distal locking screw is the incorrect placement of the target device, resulting in the creation of additional holes, into which the distal locking screw may be inserted. When the orthopedic surgeon finds this screw malposition, the screw is removed and reinserted in the correct location. However, it is difficult to predict the risk of future fractures associated with these additional holes after screw removal. Therefore, to determine the risk of fracture around additional screw holes, this study aimed to investigate the stresses formed around these holes with respect to the direction of screw penetration and the degree of osteoporosis. To this end, the authors used finite element analysis (FEA) to calculate the compression, tension, and load limits around additional transcortical-type screw holes and compared the results to those of mechanical tests using the saw bones of intertrochanteric fracture models.

\section{Methods}

\section{Three-dimensional modeling of the femur and implant}

Computed tomography (CT) scanning with a 1-mm slice thickness was performed on the femur of a healthy, 175-cm tall, 21-year-old man. A solid model of the femur was reconstructed using 3D-DOCTOR software (Able Software Corporation, Lexington, MA) to detect the boundary edge in each slice and Rapidform-TM software (INUS Technology, Inc., Seoul, Korea) to stack the slices and convert the images into an Initial Graphics Exchange Specification-type model. The dimensions were then keyed into a computeraided design (CAD) program (CATIA 2016, Dassault, France) for the reconstruction of the threedimensional models. The geometrical dimensions of the proximal femoral nail antirotation (PFNA)-II (length of $170 \mathrm{~mm}$, diameter of $10 \mathrm{~mm}, 5^{\circ}$ proximal lateralization angle, helical blade length of $95 \mathrm{~mm}$, caput-collum-diaphyseal angle of $130^{\circ}$ ) were obtained from the implant manufacturer's catalog. Then, a geometric model of the implants was assembled as a femur model, and the tip-apex distance was controlled within $20 \mathrm{~mm}$. Assuming that additional holes were formed in the anterior and posterior areas, three models were created by making additional holes with the same diameter as the distal locking screw (transcortical type) through a tangent line of the outer margin and the inner side of the cortex (additional anterior hole [AH], additional posterior hole [PH], and no additional hole [NHO] models) (Fig. 1A). The geometric model of the femur and internal fixation were imported into the FEA pre-processing software Hypermesh 18.0 (Altair, USA) to draw the mesh (Fig. 1B). After the convergence measurement, the mesh size was determined to be $1 \mathrm{~mm}$. FEA was performed using MSC-Marc 18.0 (MSC software, Inc., USA).

\section{Material properties}

All materials were assumed to be linearly elastic, isotropic, and homogeneous [3]. PFNA-Il was composed of a titanium alloy. The material properties of the bony models were obtained from the literature $[4,5]$. The cortical and cancellous bones were assumed to have elastic moduli of $16.7 \mathrm{GPa}$ and $279 \mathrm{MPa}$, respectively, and Poisson's ratios of 0.3 and 0.3 , respectively, were used for patients aged 20 years.

\section{Boundary and loading conditions}


A repetitive axial compressive force was applied at the femoral head through a rigid cylindrical body, while the bone was rigidly fixed $20 \mathrm{~mm}$ proximal from the distal end of the femur (Fig. 1C). The axial stiffness based on the finite element (FE) computation was $0.54 \mathrm{kN} / \mathrm{mm}$ and was within the measurement interval $(0.76 \pm 0.26 \mathrm{kN} / \mathrm{mm})$ [6]. The individual differences revealed that the FE model was satisfactorily validated. The boundary condition was set by fixing the distal end of the femur, and the displacement along the $x-, y$-, and $z$-axes at that site was set to zero [7]. This study simulated the forces acting on the hip during the stance phase of walking [8]. A 3000-N vertical load was applied to the femoral head [9, 10] (Fig. 1B, C). The friction coefficient was 0.3 for each bone-implant interaction [11] and implant-implant interaction [12].

\section{Observation index}

To evaluate the failure of the model, the maximum stress values under von Mises stresses were calculated around the distal locking screw at each load to determine the presence or absence of additional holes and the direction of additional holes. Furthermore, maximum stress values under Comp 11 stresses were calculated to evaluate the possibility of model failure in the tensile or compressive direction. Comp 11 stress was normalized based on a tensile strength of $135 \mathrm{MPa}$ and a compression of $200 \mathrm{MPa}$.

\section{Biomechanical experiments}

This study used a composite bone femur model (Sawbone, Pacific Research Laboratories Inc., WA, USA), an experimental artificial bone widely used in biomechanical studies. The cancellous foam core of the femur model has a density of 17 pounds per cubic foot (density per the Standard Test Method for Apparent Density of Rigid Cellular Plastics (ASTM D1622) was 0.27 with a volume fraction of 0.23), a 12$\mathrm{mm}$ canal, and an overall length of $39.1 \mathrm{~cm}$. The PFN system includes the PFNA-II (nail length $170 \mathrm{~cm}$, diameter $10 \mathrm{~cm}$, helical blade $100 \mathrm{~cm}$ ) and a distal locking screw with a diameter of $4.9 \mathrm{~mm}$. The PFNA-II, including the distal locking screw (4.9 mm diameter, $38 \mathrm{~mm}$ length), was inserted into 30 trochanteric saw bones to form 31A-21 fractures based on the Osteosynthesefragen/Orthopaedic Trauma Association classification. An additional transcortical-type hole $(4.9 \mathrm{~mm}$ ) was made anterior (AH model, 10 cases) or posterior ( $\mathrm{PH}$ model, 10 cases) to the distal locking screw at the same location (Fig. 21-E). For comparison with additional hole cases, no additional hole cases (NHO model, 10 cases) were retained. All procedures were performed by a single orthopedic surgeon with more than ten years of surgical experience.

\section{Biomechanical experimental methods}

Bone models were mounted at $10^{\circ}$ adduction and $11^{\circ}$ extension, representing the physiological direction of the maximum applied hip forces during gait, using an MTS 858 Material Testing Machine (MTS Systems Corp., Eden Prairie, MN, USA) [13]. The femoral head was seated in a customary inverted hemispherical chamber attached to a multidirectional bearing plate fixed to the load cell on the actuator of the MTS. The specimens were then cyclically loaded to failure at a frequency of $2 \mathrm{~Hz}$. Cyclic sinusoidal compressive loading with a tapered sine waveform began with a 50-500 N load stepwise, increasing 
upper loads by $50 \mathrm{~N}$ every 2500 cycles, with a maximum applied load of $50-3,000 \mathrm{~N}$. Failure was defined as construct breakage around the distal locking screw (Fig. 2F). Fixation loss or displacement of the intertrochanteric fracture was not considered a failure. The peak/valley displacements and loads for each cycle were obtained. The failure cycle $(n)$, maximum load $(\mathrm{N})$, and dynamic stiffness $(\mathrm{N} / \mathrm{mm})$ were imputed using these values.

\section{Statistical analysis}

Comparisons between the $\mathrm{NHO}$ group and the $\mathrm{AH}$ and $\mathrm{PH}$ groups were performed using Fisher's exact test. Survival analysis was performed by considering the cycle information as time and the failure case as the end point. The log-rank test was used to compare survival curves. Statistical significance was defined as a P-value< 0.05. All analyses were performed using R 4.0.1 (R Development Core Team; R Foundation for Statistical Computing, Vienna, Austria).

\section{Results}

\section{FEA of the stress changes in the cortical bone around the additional screw hole}

\section{von Mises stress distribution}

The von Mises peak stress around the distal locking screw of the NHO model was greater than the tensile strength from $2500 \mathrm{~N}$, and local breakage might have developed due to local stress concentration around the distal locking screw; however, this was difficult to visualize in cases with complete breakage (Fig. 3A). Nonetheless, the von Mises peak stress around the distal locking screw in the AH (Fig. 3B) and PH (Fig. 3C) models was higher than the tensile strength from $2000 \mathrm{~N}$; therefore, complete breakage may occur due to local stress concentration around the distal locking screw The area around the distal locking screw was structurally weaker when there was an additional hole than when there was no additional hole.

This can be explained as follows. The motion direction is attributed to the compressive force, and the stress is concentrated in the additional hole by the compressive load, increasing the possibility of femur fracture (Fig. 3D).

\section{Tensile and compressive stress distribution under Comp 11 stress}

When comparing the medial and lateral sides of the femoral bone in each model under Comp 11 stress (stress in the $x$-axis direction) subjected to the medial compression force of the femoral bone and the lateral tensile force, the maximum stress of the NHO model was $190.7 \mathrm{MPa}$. Conceivably, the medial side of the $\mathrm{NHO}$ model is not expected to be breakage by compressive load and micro breakage is possible around the distal locking screw on the lateral side however, the possibility of entire breakage in the femur was thought to be low (Fig. 4A). However, in the $\mathrm{AH}$ and $\mathrm{PH}$ models, compression on the medial side and 
tensile force on the lateral side of the femur resulted in a high stress concentration around the additional hole. Compared to the $\mathrm{NHO}$ model, the possibility of femoral fracture around the additional hole was higher, and structural vulnerability was estimated (Fig. 4B, C). In particular, at $2750 \mathrm{~N}$, the maximum stress values of $\mathrm{AH}$ and $\mathrm{PH}$ on the medial side of the femur were $238.8 \mathrm{MPa}$ and $265 \mathrm{MPa}$, respectively. The AH model showed a wider distribution of stress above the compressive strength of the femur than the $\mathrm{PH}$ model did. These results suggest that the $\mathrm{AH}$ model is at a high risk of breakage, making it more vulnerable to fracture than the PH model (Fig. 4D).

\section{Load repeat experiment in $\mathrm{NHO}, \mathrm{AH}$, and $\mathrm{PH}$ models}

Fracture around the distal locking screw, which was defined as failure (Fig. 5A), was not found in the NHO group, occurred in ten cases in the $\mathrm{AH}$ group, and in nine cases in the $\mathrm{PH}$ group. Among the ten cases of failure in the AH group, the average maximum load was $2195.8 \mathrm{~N}(1750-2650 \mathrm{~N})$, and the average cycle of load repetition was 86343.7 (64254-109500) (Table 1). Among the nine cases of failure in the PH group, the average maximum load was $2363.6 \mathrm{~N}(1800-2750 \mathrm{~N})$, and the average cycle of load repetition was 94748.4 (67323-114012). A significant difference was found in the maximum load and cycle $(\mathrm{P}<$ 0.001 ) between the $\mathrm{NHO}$ group and the $\mathrm{AH}$ and $\mathrm{PH}$ groups in Fisher's exact test. The log rank test, which defined the cycle value as time and failure as the end point, showed significant differences between the $\mathrm{NHO}$ group and the $\mathrm{AH}$ and $\mathrm{PH}$ groups $(\mathrm{P}<0.001)$ (Fig. 5B). Between the $\mathrm{AH}$ and $\mathrm{PH}$ groups, neither Fisher's exact test $(P=1)$ nor the log rank test $(P=0.072)$ showed significant differences (Fig. $5 C$ ).

Table 1

The results of repetitive-load biomechanical testing

\begin{tabular}{|llll|}
\hline Type $(\mathbf{n}=10)$ & Fail & Cycle to fail $(\min -$ max $)$ & Newton $(\mathbf{N})$ to fail $($ min-max $)$ \\
\hline Model NHO & 0 & N/A & N/A \\
\hline Model AH & 10 & $86343.7(64254-109500)$ & $2195.8(1750-2650)$ \\
\hline Model PH & 9 & $94748.4(67323-114012)$ & $2363.6(1800-2750)$ \\
\hline $\begin{array}{l}\text { NHO, no-hole model; } \mathrm{AH} \text {, anterior additional hole model; } \mathrm{PH}, \text { posterior additional hole model; N/A, not } \\
\text { applicable }\end{array}$ & & \\
\hline
\end{tabular}

\section{Discussion}

Despite the development of implants, devices, and surgical techniques, complications related to the distal locking screw of the PFN system remain [2]. These include insufficient dissection of the fascia, excessive tightening of the distal locking screw, and loosening of the targeting device between the nails [14]. The most common initial complication is unstable coupling. When a distal locking screw is inserted in an incorrect position, it creates an additional hole at the anterior or posterior femur.

The following reasons may explain possible occurrence of fracture around the hole. First, fractures occur because of cortical bone loss caused by an additional hole. The cortical bone is critical for maintaining the mechanical strength of the bone. In particular, a 3-mm hole in the cortical long bone decreases the 
bending strength by $40 \%$, while the torsional strength reduces by approximately $12 \%$ [15]. However, the PFNA-Il uses a 4.9-mm screw, and it is expected that these decreases in bending and torsional strength are even greater. Therefore, the authors estimated that repeated loads may induce fractures when such a large amount of cortical bone loss negatively affects the mechanical strength of the femur.

Second, the type of additional holes may affect the incidence of fracture. Additional screw holes can be classified into unicortical, bicortical, half-bicortical, and transcortical penetrations [16]. Most additional screw holes that are directed anteriorly and posteriorly to avoid nails are of the transcortical type. It is supposed that the highest stresses result from transcortical penetration, and the fracture risk ratio was dramatically elevated under both axial and torsional loads [17]. If an additional screw hole occurs in a patient with a large proportion of the nail in the medullary cavity, such as in an Asian with a small body frame, the hole follows a transcortical shape and is pushed outward from the center by the nail, which can generate a risk condition for fracture. In addition, thermal bone necrosis that occurs during transcortical penetration may further increase the risk of fracture [18].

Third, the stress concentration around the additional holes may influence the occurrence of fractures. The standard walking condition results in the bending of the bone during the stance phase. This generates tensile stress in the lateral femur [19]. The peak stress concentration of the femurs in trochanteric fractures was mainly located in the site where the distal lock screw was making contact [20]. Robinson et al. [21] suggested that cortical bone hypertrophy around the distal nail, observed on a simple radiograph during the healing process of intertrochanteric fractures using an intramedullary nail, was a radioactive hallmark, suggesting that stress concentration occurred around the distal nail [21]. Stress concentration can reduce the mechanical integrity of a bone, making it more susceptible to sudden brittle fracture during trauma or to gradual fatigue failure over time (stress fractures). A distally locked construct bears most of the load, which is gradually transferred to the distal cortex as the fracture heals. With good cortical apposition of the fracture, the bone cortices support most of the compressive load. Without cortical contact of the fracture, the entire load is transferred to the distal screw through the nail until the fracture heals [22]. Therefore, efforts should be made to increase the stable cortical bone contact surface of the fracture through possible anatomical reduction before nail insertion.

Fourth, the mechanical influence should be considered. Additional holes allow the movement of the nail at its junction in the medulla. This movement may cause the nail to slip anteriorly or posteriorly and lead to the eccentric position of the nail in the medullary canal, which will direct mechanical stimulation to the anterior or posterior side of the femur and may cause a fracture [23, 24]. In particular, Asian women have shorter femoral necks and smaller femoral neck angles and increased anterior bowing of the shaft than Western women do. Therefore, the nail could be located eccentrically in the intramedullary space [25], leading to a higher risk of fracture. Notably, biomechanical loading tests and FEA studies have shown that $\mathrm{AH}$ was more susceptible to fracture than $\mathrm{PH}$. The femur with anterior bowing is subjected to increased posteromedial compressive force and anterolateral tensile force. Considering its physical properties, which are more vulnerable to tensile strength, the bone may be more susceptible to fracture 
when an $\mathrm{AH}$ is formed. Therefore, if an $\mathrm{AH}$ occurs in older patients with osteoporosis and severe bowing, the risk of fracture would be further increased.

This study had some limitations. First, this study did not consider various forms of load from the human body and did not use real human bones. The authors used a composite femoral bone model to overcome this problem, determined ten identical conditions for each experimental condition, investigated the stress change according to the load using FEA, and further investigated the effect of bone density. Second, the study did not reflect various fracture types and reduction statuses. The authors applied a fracture model with the highest severity among fracture types, excluding reverse oblique intertrochanteric fracture for which the prognosis is known to be poor. The experiment was conducted assuming that stable reduction was obtained. Third, various types of additional holes, such as unicortical, bicortical, half-bicortical, and transcortical penetrations, were not tested. However, the authors chose the transcortical type to focus on in this study because this type is the most common and the most susceptible to fracture. Finally, the effects of the various diameters of the medulla and nail and the position of the distal end of the intramedullary nail were not considered, and various designs of the PFN system were not tested. Future biomechanical studies incorporating many cadaveric bone models under various conditions and nail systems are recommended.

\section{Conclusion}

During surgery for intertrochanteric fracture using the PFN system, when the distal locking screw is malpositioned anterior and posterior to the nail, the remaining hole may be a risk factor for fractures around it once removed. Therefore, the surgeon should pay great attention during the operation to prevent accidental occurrence of additional screw holes.

\section{Abbreviations}

Anterior hole (AH)

Computed tomography (CT)

Computer-aided design (CAD)

Finite element analysis (FEA)

Material Testing Machine (MTS)

No additional hole (NHO)

Posterior hole (PH)

Proximal femoral nail (PFN) 


\section{Declarations}

Ethics approval and consent to participate: Not applicable.

The design and protocol of this study were approved by the institutional review board of the corresponding author's hospital (GJVH-IRB No. 2020-8-3). All patients were informed that their medical data could be used in a scientific study, and they provided consent.

All experiments and methods were performed in accordance with relevant guidelines and regulations of by the institutional review board of the a corresponding author's hospital(GJVH-IRB). All experimental protocols were approved by the institutional review board of the a corresponding author's hospital(GJVHIRB).

Consent for publication: Not applicable.

Competing interests: The authors declare that there are no conflicts of interest.

Acknowledgements: This study was supported by a VHS Medical Center Research Grant, Republic of Korea(VHSMC 21011).

Funding: This research did not receive any specific grant from funding agencies in the public, commercial, or not-for-profit sectors.

Authors' contributions: $\mathrm{CHM}$ and CSM conceived and coordinated the study. CHM designed the ethical issues. CHM and PJ acquired the data. CHM, CSM, and BJH reviewed the data. $\mathrm{YL}$ and CHM performed the statistical analysis. HMC, JP, and BJH prepared the manuscript. JP and CSM provided administrative, technical, and material support. All authors approved the final manuscript.

Availability of data and materials: The datasets during and/or analysed during the current study available from the corresponding author on reasonable request.

\section{References}

1. Skála-Rosenbaum J, Bartonícek J, Bartoška R. Is distal locking with IMHN necessary in every pertrochanteric fracture? Int Orthop. 2010;34:1041-7.

2. Gadegone WM, Salphale YS. Proximal femoral nail: an analysis of 100 cases of proximal femoral fractures with an average follow up of 1 year. Int Orthop. 2007;31:403-8.

3. Henschel J, Eberle S, Augat P. Load distribution between cephalic screws in a dual lag screw trochanteric nail. J Orthop Surg Res. 2016;11:41.

4. McCalden RW, McGeough JA, Barker MB, Court-Brown CM. Age-related changes in the tensile properties of cortical bone. The relative importance of changes in porosity, mineralization, and 
microstructure. J Bone Joint Surg Am. 1993;75:1193-205.

5. McCalden RW, McGeough JA, Court-Brown CM. Age-related changes in the compressive strength of cancellous bone. The relative importance of changes in density and trabecular architecture. J Bone Joint Surg Am. 1997 Mar 79(3):421-7.

6. Papini M, Zdero R, Schemitsch EH, Zalzal P. The biomechanics of human femurs in axial and torsional loading: comparison of finite element analysis, human cadaveric femurs, and synthetic femurs. J Biomech Eng. 2007;129:12-9.

7. Lee $\mathrm{CH}$, Su KC, Chen KH, Pan CC, Wu YC. Impact of tip-apex distance and femoral head lag screw position on treatment outcomes of unstable intertrochanteric fractures using cephalomedullary nails. J Int Med Res. 2018;46:2128-40.

8. Bergmann G, Deuretzbacher G, Heller M, Graichen F, Rohlmann A, Strauss J, et al. Hip contact forces and gait patterns from routine activities. J Biomech. 2001;34:859-71.

9. Rohlmann A, Mössner U, Bergmann G, Kölbel R. Finite-element-analysis and experimental investigation of stresses in a femur. J Biomed Eng. 1982;4:241-6.

10. Chen WP, Tai CL, Shih CH, Hsieh PH, Leou MC, Lee MS. Selection of fixation devices in proximal femur rotational osteotomy: clinical complications and finite element analysis. Clin Biomech (Bristol, Avon). 2004;19:255-62.

11. Li J, Zhao Z, Yin P, Zhang L, Tang P. Comparison of three different internal fixation implants in treatment of femoral neck fracture a finite element analysis. J Orthop Surg Res. 2019;14:76.

12. Kim JT, Jung $\mathrm{CH}$, Shen QH, Cha YH, Park CH, Yoo Jl, et al. Mechanical effect of different implant CCD angles on the fracture surface after fixation of an unstable intertrochanteric fracture: a finite element analysis. Asian J Surg. 2019;42(11):947-56.

13. Schwachmeyer V, Damm P, Bender A, Dymke J, Graichen F, Bergmann G. In vivo hip joint loading during post- operative physiotherapeutic exercises. PLoS One. 2013;8(10):e77807.

14. Hesse B, Gächter A. Complications following the treatment of trochanteric fractures with the gamma nail. Arch Orthop Trauma Surg. 2004;124(10):692-8.

15. Laurence M, Freeman MA, Swanson SA. Engineering considerations in the internal fixation of fractures of the tibial shaft. Bone Joint Surg Br. 1969;51(4):754-68.

16. Jung HJ, Jung YB, Song KS, Park SJ, Lee JS. Fractures associated with computer-navigated total knee arthroplasty. A report of two cases. J Bone Joint Surg Am. 2007;89(10):2280-4.

17. Kim K, Kim YH, Park WM, Rhyu KH. Stress concentration near pin holes associated with fracture risk after computer navigated total knee arthroplasty. Comput Aided Surg. 2010;15(4-6):98-103.

18. Ho KW, Gilbody J, Jameson T, Miles AW. The effect of $4 \mathrm{~mm}$ bicortical drill hole defect on bone strength in a pig femur model. Arch Orthop Trauma Surg. 2010;130(6):797-802.

19. Fox MJ, Scarvell JM, Smith PN, Kalyanasundaram S, Stachurski ZH. Lateral drill holes decrease strength of the femur: an observational study using finite element and experimental analyses. J Orthop Surg Res. 2013;8:29. 
20. Huang X, Yu B, Gu Y, Li Z. Biomechanical comparison of dynamic hip screw and gamma nail for the treatment of unstable trochanteric fractures: A finite element study. Int J Clin Exp Med. 2017;10:7867-74.

21. Robinson $\mathrm{CM}$, Adams $\mathrm{Cl}$, Craig M, Doward W, Clarke MC, Auld J. Implant-related fractures of the femur following hip fracture surgery. J Bone Joint Surg Am. 2002;84:1116-22.

22. Koyuncu Ş, Altay T, Kayalı C, Ozan F, Yamak K. Mechanical failures after fixation with proximal femoral nail and risk factors. Clin Interv Aging. 2015;10:1959-65.

23. Lacroix $H$, Arwert $H$, Snijders CJ, Fontijne WP. Prevention of fracture at the distal locking site of the gamma nail. A biomechanical study. J Bone Joint Surg Br. 1995;77:274-6.

24. Shen WY. Complications with the gamma nail and long gamma nail, and their prevention. Osteo Trauma Care. 2005;13:34-41.

25. Chang SM, Song DL, Ma Z, Tao YL, Chen WL, Zhang LZ, Wang X. Mismatch of the short straight cephalomedullary nail (PFNA-II) with the anterior bow of the femur in an Asian population. J Orthop Trauma. 2014;28:17-22.

\section{Figures}




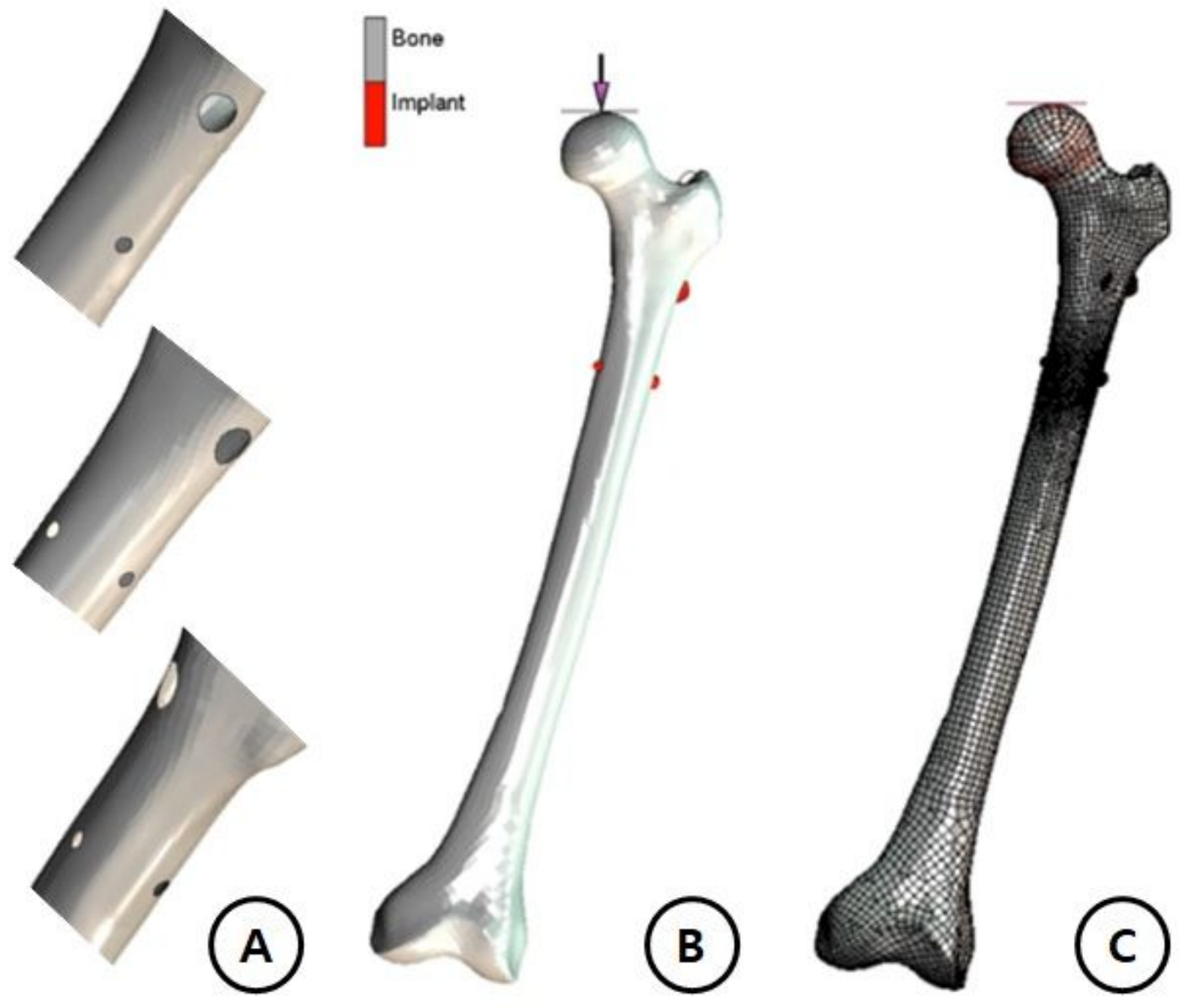

Figure 1

(A) Comparison of stress distribution among three cases according to nail screw hole locations. (B) Creating a rigid body to simulate the experimental conditions of the upper part of the femur, transferring the load to the head of the femur $(0-3,000 \mathrm{~N})$, and setting the contact glue to the rigid body (cylinder) and the femoral head. (C) The femur is made in three parts of three-dimensional shell modeling, and the Implant (PFNA) is made using Tetra/Hexa Mesh modeling.

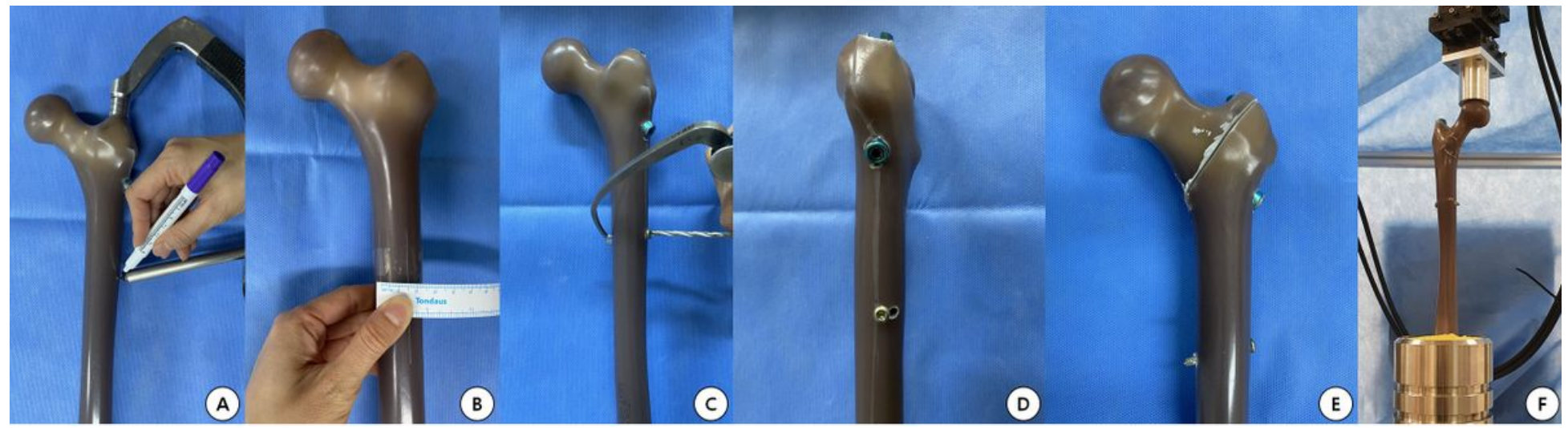




\section{Figure 2}

Creating an experimental model of a composite femur bone. (A) Making the start point (SP) using the target device. (B) If the distal locking screw is fixed from the SP and additional holes are formed in the front or rear, the insertion point and exit point are identified. (C) To generate anterior or posterior additional holes or correct hole for distal targeting screw, the holes are drilled using a 4.0-mm drill bite and a 4.9-mm screw is inserted/removed using anterior cruciate ligament reconstruction guide. (D) Model with distal locking screw fixed in the correct position using posterior additional screw hole. (E) All bone model fractures are formed based on Osteosynthesefragen/Orthopaedic Trauma Association classification into trochanteric 31A-21. (F) The femoral head is seated into a customary inverted hemispherical chamber attached to a multidirectional bearing plate fixed to the load cell on the actuator of the material testing machine and then cyclically loaded to failure.
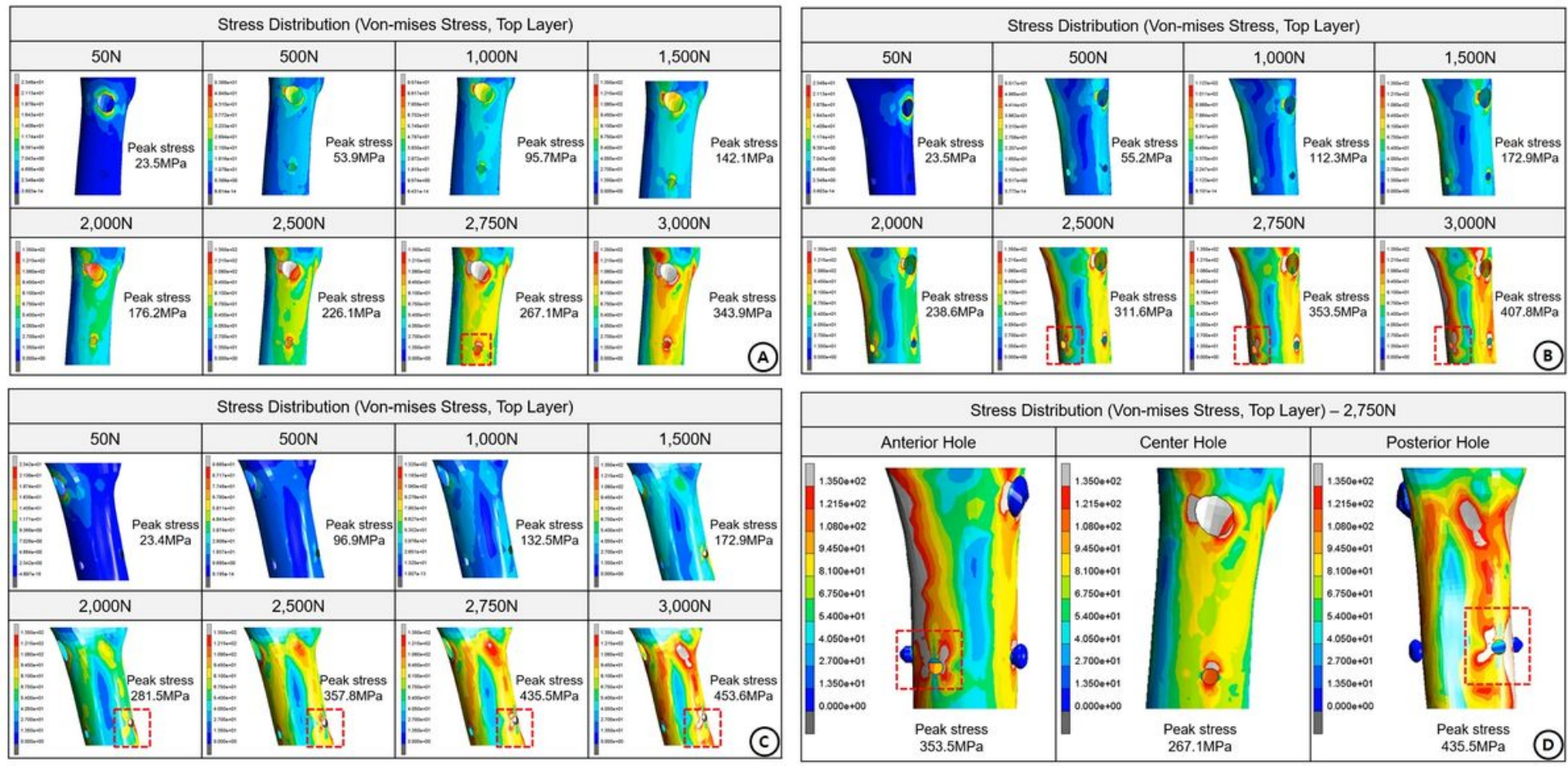

\section{Figure 3}

(A) Center hole case. The stress around the hole occurs over the tensile strength (135 MPa) from 2,500 N, and local damage may occur because the stress is usually concentrated around the hole. Stress distribution (von Mises stress, top layer). (B) Anterior hole case. Tensile strength (135 MPa) or higher stress occurs from 2,000 $\mathrm{N}$ of load around the hole. Stress concentration occurs around the anterior additional hole. There is a high possibility of breakage. Stress distribution (von Mises stress, top layer). (C) Posterior hole case. Tensile strength (135 MPa) or higher stress occurs from the load around the hole from 2,000 N. Stress concentration around the posterior additional hole occurs. Similar to the anterior case, there is a high probability of breakage. Stress distribution (von Mises stress, top layer). (D) Von mises stress. Tensile strength (135 MPa) or higher stress concentration occurs around the additional 
holes. This suggests a high possibility of damage by compressive load stress distribution (von Mises stress, top layer) at $2,750 \mathrm{~N}$.
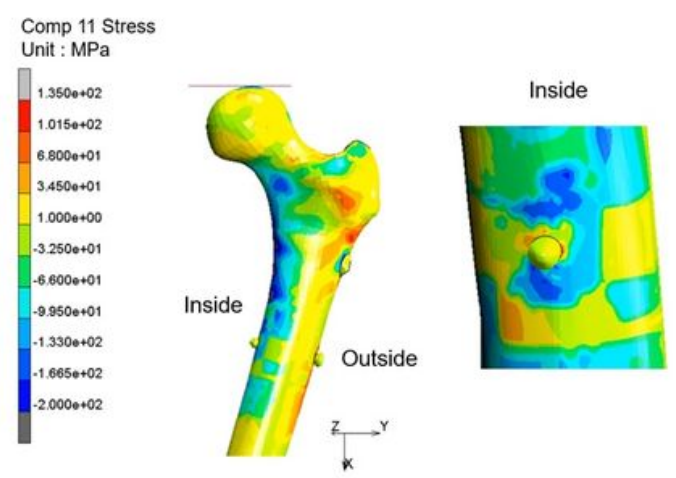
Comp 11 Stress
Unit: $\mathrm{MPa}$
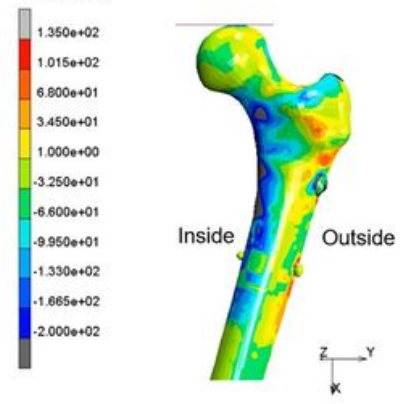

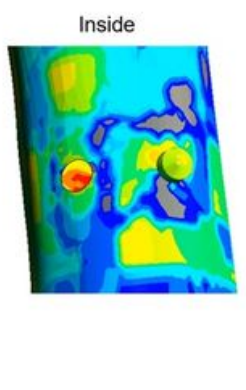

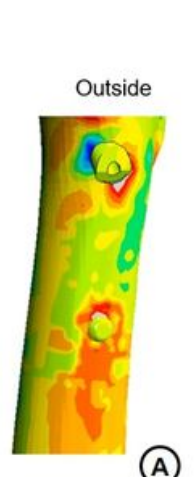
Comp 11 Stress Unit : MPa
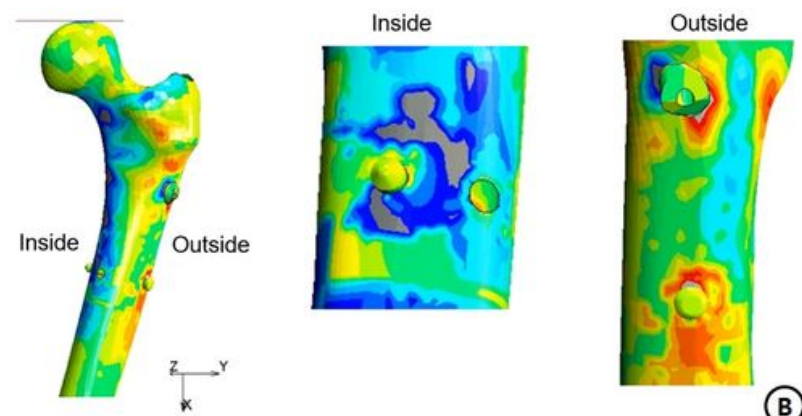

(B)
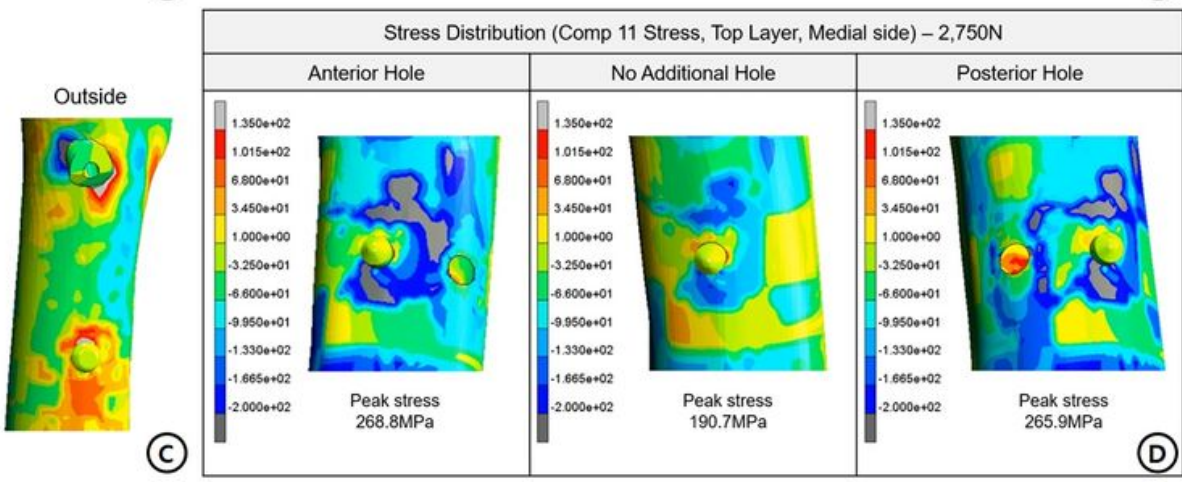

Figure 4

(A) Comp 11 stress (stress in the $x$-axis direction). Stress generation by tensile stress around the medial side of the femur and compressive stress around the lateral side of the femur (compressive stress, 200 $\mathrm{MPa}$ ). The possibility of damage to the medial side is low and that to the lateral side is also low, except for minute damage around the hole. (B) Comp 11 stress anterior hole case. Under stress distribution, at $2,750 \mathrm{~N}$, there is a high possibility of damage around the additional hole due to the medial compression force and lateral tensile force, because of structural weakness and high possibility of damage caused by cracking from the additional hole toward the outer hole. (C) Comp 11 stress posterior hole case. Under stress distribution, at $2,750 \mathrm{~N}$, there is a high possibility of damage around the additional hole due to the medial compression force, lateral tensile force, and structural weakness. (D) Center hole case. The possibility of damage is low around the medial side. However, breakage is highly possible when an additional hole occurs. The anterior hole case is the most vulnerable because the maximum stress is higher than that in the posterior hole case and the damaged area is wider. 

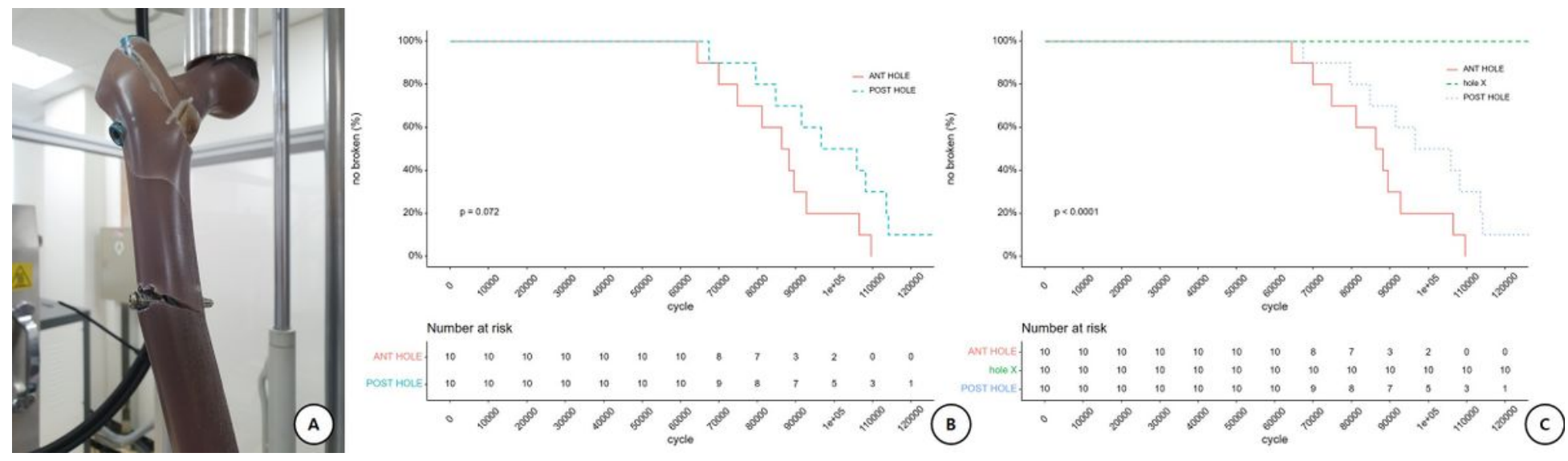

Figure 5

(A) Failure is defined as construct breakage around the distal locking screw, whichever occurred first (B) Log rank test results, which defined cycle value as time and failure as end point, show significant difference between the $\mathrm{NHO}$ group and $\mathrm{AH}$ and $\mathrm{PH}$ groups $(\mathrm{P}<0.001)$. (C) Between $\mathrm{AH}$ group and $\mathrm{PH}$ group, neither Fisher's exact test $(P=1)$ nor Log rank test $(P=0.072)$ showed significant difference. 